\title{
Simulation, Modeling, and Evaluation of Satellite-Based Multicasting Protocols
}

\author{
Ryan W. Thomas, Richard A. Raines, Rusty O. Baldwin, Michael A. Temple \\ Department of Electrical and Computer Engineering \\ Air Force Institute of Technology \\ 2950 P Street \\ Wright Patterson AFB, OH 45433
}

\begin{abstract}
Satellite-based mobile multicasting is a largely unexplored and untested area of networking. This paper examines the performance associated with applying Distance Vector Multicast Routing Protocol (DVMRP) and On Demand Multicast Routing Protocol (ODMRP) to a six plane, 66-satellite low earth orbit satellite (LEOs) constellation. ODMRP provides high Quality-of-Service performance at the expense of bandwidth efficiency. In contrast, DVMRP is over twice as efficient, with only a $10 \%$ lower Quality-of-Service. Both protocols are stressed under a single satellite failure. For DVMRP, this leads to greater end-to-end delay and slightly higher efficiency. ODMRP shows no significant change from the non-failure case.
\end{abstract}

\section{INTRODUCTION}

Transmitting multicast information through a dynamic global satellite network is more complex than its terrestrial equivalent. Whereas in the wired terrestrial environment, the user's location and network topology is relatively fixed, in the wireless satellite-based environment, the locations of the multicast members are constantly changing and the network topology is no longer certain. This paper examines two different terrestrial protocols for low earth orbit satellite (LEOs) multicast communications. The On Demand Multicast Routing Protocol (ODMRP) and Distance Vector Multicast Protocol (DVMRP) are investigated and evaluated in light of the special challenges associated with LEO satellite communications.

\section{A. Mobile IP}

Mobile IP is the primary method of mobility management for terrestrial IP networks. It is designed to deal with mobile networks operating on the fringe of a fixed network. Mobile IP assumes that this fixed network has the capacity to route or tunnel IP packets across it. Furthermore, being stacked between the transport layer and the IP network layer in the OSI model, it is not concerned with how these routing mechanisms work. Mobile IP is only designed to maintain a nomadic node's connection with its fixed home Internet location.

Mobile IP is described in RFC 2002 through 2006 $[1][2][3][4][5]$, and collectively these documents present a system that allows nodes to maintain a permanent IP address wherever they go. To accomplish this, there are three primary players in Mobile IP [1]:
The mobile node: This is a node that has the ability to change the link used to connect into the network and still maintain communication over a permanent IP address.

The home agent: This is a router that can access the mobile node's home link as determined by the mobile node's permanent IP address. The home agent is responsible for:

- Knowing the mobile node's current location,

- Advertising its reachability in the mobile node's absence, and

- Intercepting and forwarding packets destined to the mobile node to the mobile node's care-of-address

Foreign agent: This is a router that can access the mobile nodes current link. It is responsible for:

- Helping the mobile node alert its home agent of it's care-of-address,

- If necessary, providing a care-of-address, receive packets from the home agent, and send them to the mobile node, and

- Routing packets generated by the mobile node to their appropriate destinations.

The care-of-address mentioned repeatedly in the agent roles is just what it sounds like. Like a vacationing tourist might have his or her mail forwarded by a servant to a temporary address where he or she is staying, mobile nodes have their traffic forwarded to them via a care-of-address. The three players' responsibilities are centered on negotiating for the care-of-addresses and then using them.

There are two documented mechanisms for performing mobility management under Mobile IP: co-located and tunneled. Co-located involves forwarding data directly to a mobile node through a foreign agent, whereas tunneling sends data from source to home agent to foreign agent to mobile node. To eliminate Mobile IP's habit of "doglegging" (the routing effect that occurs when a message is tunneled away from the most efficient route because of its need to go through an agent), the co-located approach was used.

Mobile IP does not dictate the multicast routing mechanism to be used. Distance Vector Multicast Routing Protocol (DVMRP) is the oldest and most implemented multicast mechanism, so it is a fit for the mobile IP paradigm. DVMRP was derived from the Routing Information Protocol (RIP); a unicast method of routing that calculates the best next hop to use to transmit a packet to a destination. DVMRP

The views expressed in this article are those of the author and do not reflect the official policy of the United States Air Force, Department of Defense or the U.S. Government

U.S. Government work not protected by

U.S. copyright 
uses a source-based multicast tree to pass packets to their destinations. When a source wishes to send a multicast message, the network is flooded with the first packet in such a way as to form a tree. The routers then "prune" off unnecessary branches (i.e., those that have no receivers on them) of the tree [6]. This technique of flooding and pruning is called Reverse Path Forwarding (RPF) and is the key to designing source-based trees without any a priori network state knowledge.

The flooding part of the RPF algorithm works as follows: when a node receives a packet, the DVMRP routing table is used to determine if the link that the packet came in on is the best way to get to the source node. If it is, then the node is forwarded on all out-going links except the one it arrived on. If it isn't, then the packet is destroyed. This ensures the tree only contains the shortest paths from source to destination [6]. Next, the Internet Group Management Protocol (IGMP) is used to prune branches by querying to see if there are any subscribers on each of the branches. If there are no subscribers, a prune command is sent to the upstream router, instructing it not to forward multicast packets to this branch. If all branches leaving from a particular node have been pruned, that node forwards a prune message down the tree from itself, completely cutting itself off from the group. If a node on a previously pruned branch decides to subscribe to a multicast group, it is "grafted" on by sending a cancel-prune message to the upstream nodes. This allows for a tree to change without completely starting the process over.

Periodically, however, the tree may need to be refreshed because of changes in the topology and membership. The refresh mechanism involves a repetition of the above process. This flood and prune technique negates some of the bandwidth benefits of multicasting. For groups with many sources, this is a significant burden because of the high network and memory utilization needed to maintain many source tree routes [7].

When a node receives a Join Reply packet, the packet is examined to see if the address is located under the next-hop field. If it is, the node goes back into its records and finds the next-hop address for that particular source. The node itself then creates a Join Reply and sends it upstream. At this point, the node becomes a forwarding node for the multicast group. A flag is set by the node to indicate it should forward this group and records the next-hop address. Like the node that requested to receive the group, this routing node groups together Join Replies before broadcasting upstream to save bandwidth. When the Join Reply message reaches the source, the source continues to use the designated route until a predefined route expiration timer elapses. The process then repeats.[10]. Note that each source will generate new routes, potentially adding to existing routes to form a mesh.

\section{B. Mobile Ad Hoc Networks (MANETs)}

Mobile ad hoc networks (MANETs) are those that have no long-term topology. These networks operate with all nodes acting as routers as well as sources and destinations of data. On Demand Multicast Routing Protocol (ODMRP) was developed primarily as an ad hoc protocol for IEEE 802.11 networks and does not, by its nature, require any external form of mobility management.

ODMF:P is a mesh-based approach to routing [9][10]. Meshes are advantageous to trees in ad hoc networks because they are able to deal with many of the problems of mobility. The capacity to handle intermittent connectivity and uneven traffic density are two of the more significant advantages. It also is an on-demand algorithm, meaning it builds routes between nodes only as required by source nodes. When a multicast source has a message to send (and there is no route determined from this source to the group yet), it sends out a request in the form of Join Query. The Join Query is made up of a table with fields. The first field is the source's IP address followed by the node address of the query's last hop. In the case of the originator, the last hop is the source IP address again. The next field is the sequence number, which is designed to differentiate packets from one another. Finally, a Time-To-Live (TTL) field is configured to prevent the message from being propagated outside the multicast domain.

The Join Query is sent to all neighbors of the source. Each neighbor records the source and sequence of the packet, as well as the last hop IP address. The node then decrements the TTL field of the packet and checks whether the field is greater than 0 . If it is, the node forwards the query to its neighbors; otherwise, the packet is destroyed. The node finally examines whether it wants to subscribe to the group. If it does, it creates and transmits a Join Reply[9].

When a node receives a Join Reply packet, the packet is examined to see if the address is located under the next-hop field. If it is, the node goes back into its records and finds the next-hop address for that particular source. The node itself then creates a Join Reply and sends it upstream. At this point, the node becomes a forwarding node for the multicast group. A flag is set by the node to indicate it should forward this group and records the next-hop address. Like the node that requested to receive the group, this routing node groups together Join Replies before broadcasting upstream to save bandwidth.

When the Join Reply message reaches the source, the source continues to use the designated route until a predefined route expiration timer elapses. The process then repeats [10]. Note that each source will generate new routes, potentially adding to existing routes to form a mesh. Lee et al. determined ODMRP has significantly lower channel and storage overhead than DVMRP as well as the capability to exploit redundant paths--something none of the other protocols allow. Additionally, future enhancements incorporating positional information may allow for more efficient route determination by decreasing the frequency of route refresh [11]. 


\section{SIMULATION}

\section{A. Experimental Design}

The satellite network simulated was an Iridium-like 6 plane, 66 satellite network. Satellites are able to communicate with co-planar satellites that are immediately adjacent over Inter-Satellite Links (ISLs). Except for the polar regions above and below the $+/-60$ degree latitude line, the satellites also are able to communicate with satellites in adjacent orbital planes. The satellite-to-satellite delay is a function of propagation time, transmission rate and queuing delay.

Earth stations are considered to be able to communicate with a satellite if the satellite is within a predefined radius. The ground stations-satellite link was modeled with multiple access capability to prevent collisions when more than one ground station was communicating with a single satellite. Additionally, earth stations were placed in seven geographically separated urban areas: Rio de Janero, Melbourne, Kansas City, Dharan, Beijing, Berlin, and Capetown.

Several factors were varied over the course of the experiment including group membership level, loading level, and transmission mechanisms. Performance was evaluated with 5,10 and 15 member groups. Loading levels, derived from the maximum throughput of an ISL, were set to 50,80 and $100 \%$. Multicast messages were sent in a one-to-all and an all-to-all transmission mechanism. Additionally, each protocol was examined under the presence of a critical satellite failure. Measuring the affect of these factors on the system performance was accomplished by using three metrics: data-to-overhead ratio, received-to-sent ratio, and end-to-end delay.

\section{B. Performance Metrics}

To determine the effectiveness of the DVMRP and the ODMRP protocols, three system performance metrics are defined. Each is described below.

A Quality-of-Service (QoS) metric was investigated by measuring the ratio of packets received to packets sent. The received-to-sent ratio is defined as the total number of packets uniquely received by all receivers divided by the total number of packets sent by all multicast sources multiplied by the number of multicast receivers.

The average end-to-end delay for the entire system was also measured to determine the overall speed of the protocols. In conjunction with the other metrics, it tracks the queue length and the route optimization. While a metric such as hop count or hop ratio (ratio of hops taken to minimal number of hops) might be useful here, the constellation is not composed of equidistant ISLs and hop count could be a misleading metric. Since the distance between satellites at the sixty-degree latitude mark is half the distance at the equator, the number of hops is not necessarily proportional to the amount of delay.

Additionally, since satellites are so far apart, the mean delay is a better indicator of the effectiveness of the routing service. Mean delay is simply the average delay of all packets received by all receivers.

To evaluate the bandwidth efficiency of the protocol, the ratio of data-to-overhead was measured on a system-wide perspective, rather than a source-based one. Unlike mean delay, this control ratio is representative of the overhead each protocol requires. As such, this metric provides insight into the other metrics by showing how much work is required to achieve the outcome. Data bits are defined to be bits that are successfully transmitted from source to receiver. Overhead bits are all other information transmitted over the system, including data bits that do not reach their destinations.

The ratio of data bits to overhead bits is examined systemwide. It is calculated by first counting the total of all bits that are transmitted in the system from any source. This is the net network traffic. Next, when packets are received at a destination, the packet id, size, and all hop IP addresses are recorded in a master list. When other multicast group destinations receive the same packet, they update only the new hops received at that location into the master list. At the end of the simulation, the size of the packet and total number of hops are combined to determine the total data bit traffic. This is subtracted from the net network traffic to determine the total overhead traffic, and a ratio is taken of the total data traffic and the total overhead traffic.

To evaluate the performance of the protocols in a nonideal network, the satellites with the highest traffic load was removed from the topology after allowing the protocol to reach a steady-state period of operation.

\section{RESULTS}

Figures 1 and 2 depict the performance metrics in the one-to-all transmission mechanisms. Inset (a) represents the bandwidth efficiency metric, inset (b) the QoS metric, and inset (c) the protocol speed.

\section{A. Quality-of-Service}

The mesh-based approach of ODMRP leads to greater than $99 \%$ packets received to those sent in the all-to-all case. However, this same mesh-based approach is also responsible for the low efficiency of the protocol. Through overredundancy, the mesh creates enough copies of the data packets to ensure delivery around any obstacles. This redundancy ensures the routes utilized for delivery will be current with respect to the most recent network topology changes.

DVMRP consistently has between 88 and $93 \%$ of all packets received. DVMRP is dependent on creating and maintaining fresh vector routing data to successfully deliver packets. If a vector becomes outdated during a packet 
transmission, the data being transmitted is lost. This is in contrast to ODMRP's capability of devising a new from its on-demand and mesh-based features. The more satellites acting as forwarding group members, the better the protocol is able to correct from unexpected route failures. (a)

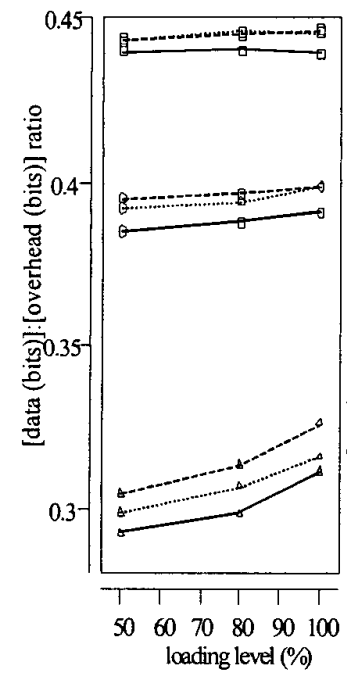

(a)

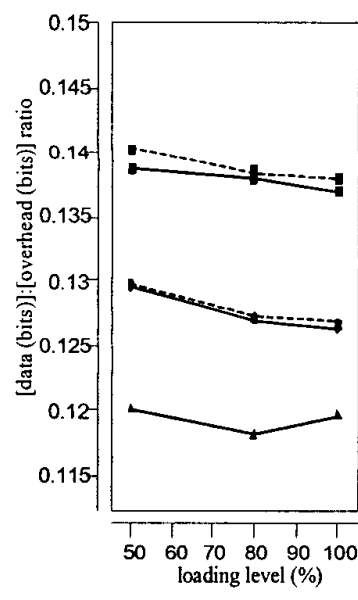

(b)

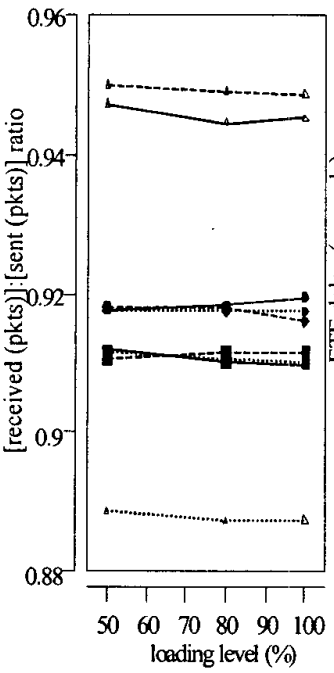

(c)

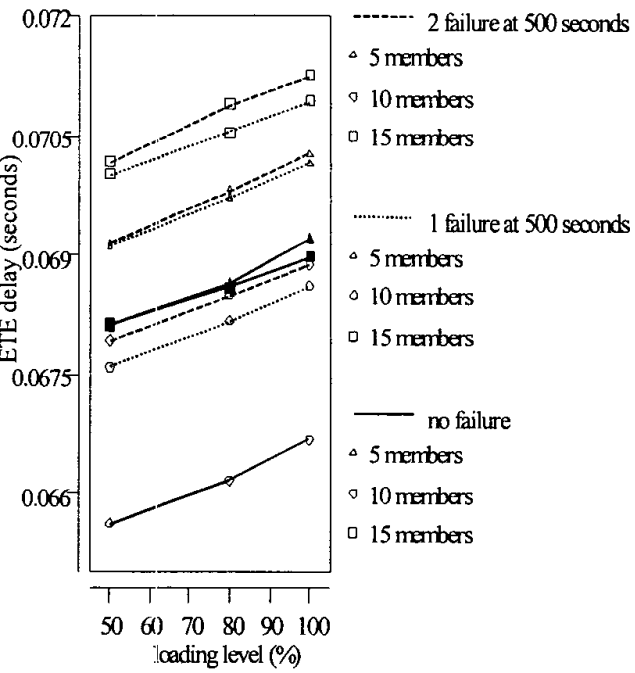

Figure 1: DVMRP One-to-All

(b)

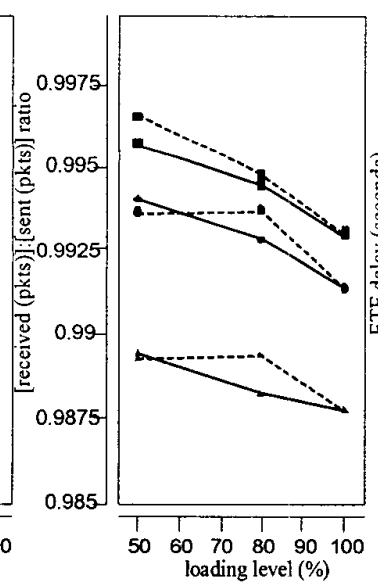

(c)

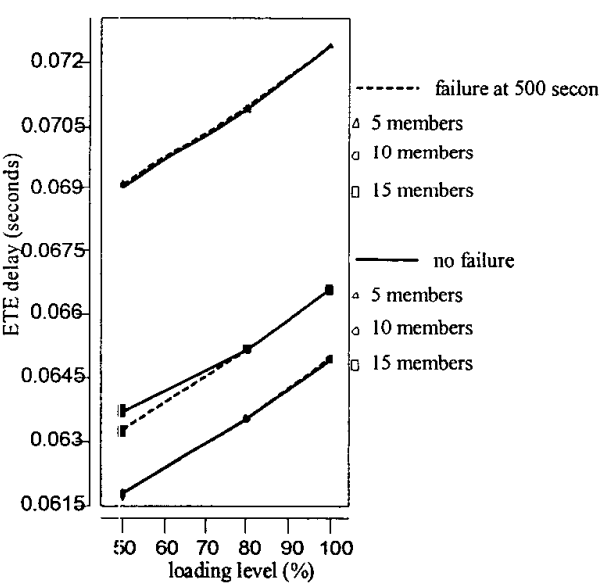

Figure 2: ODMRP One-to-All Scenarios

For both protocols, Analysis of Variance (ANOVA) indicates the mechanism of transmission (all-to-all or one-toall) creates more than $70 \%$ of the variance in the QoS metric. The variance is different for each protocol: ODMRP becomes more reliable, while DVMRP becomes less reliable in the all-to-all mode. In ODMRP, the all-to-all mode provides more forwarding group members than the one-to-all mode, creating more resilient meshes and a higher QoS.

\section{B. Speed}

In terms of data transfer speed both protocols have similar behavior. A delay difference of less than $8 \mathrm{~ms}$ was observed for all scenarios simulated.

As expected, the mean end-to-end delay increases as the loading level is increased. This is directly attributable to the queue length, since the transmission speed of the intersatellite links is a constant value. When placed under $100 \%$ 
link loading level, $\rho$ (the ratio of bit arrival rate to bit transmit rate) approaches one for certain links, creating longer delays.

However, the end-to-end delay of ODMRP is more sensitive to loading than DVMRP. ANOVA demonstrates that while $30 \%$ of the end-to-end variance of ODMRP is related to the workload, less than $1 \%$ of DVMRP's variance is workload related. This discrepancy is related to the additional queuing delay created from the mesh-based architecture. Additional sources create additional forwarding group members that forward data packets onto more ISLs, increasing delay.

\section{Bandwidth Efficiency}

The bandwidth efficiency metric reveals that the ODMRP protocol has a large amount of overhead resulting from its on-demand aspect. In contrast, much of the overhead from DVMRP is fixed, regardless of the multicast membership level and traffic intensity. By adding membership to the same urban areas, the multicast trees become more efficient, increasing the data-to-overhead ratio. Analysis of variance lends additional weight to this, as $77 \%$ of the bandwidth efficiency variance is caused by the membership factor, while $19 \%$ comes from the mechanism of transmitting (one-to-all or all-to-all).

Unlike DVMRP, the efficiency ratios associated with the all-to-all transmission scenario for ODMRP vary by less than 0.0128 from the highest to lowest ratio. The research shows ODMRP is affected more by the mechanism transmission scenario than by workload or membership, since changing from an all-to-all to a one-to-all transmission scenario more than doubled the data-to-overhead ratio.

\section{Robustness}

DVMRP has an increase in end-to-end delay from the additional routing that occurs to route direct packets around the failed satellite. Additionally, DVMRP sees a small improvement in bandwidth efficiency with the loss of a satellite, caused by the slight decrease in flood and prune required to map out the smaller, damaged network. In contrast, ODMRP has no statistical difference between the failed and non-failed scenario.

The largest affect the failed satellite has on both protocols occurs when the presence of a dead satellite directly above a ground station prevents it from sending or receiving packets, and reduces the received-to-sent ratio. However, this is not a condition of the routing protocol, but rather the connectivity of the network. For fully-connected networks, the difference between the damaged network performance and the non-damaged network is minimal.

\section{CONCLUSIONS}

Both DVMRP and ODMRP have strengths. For use of bandwidth, the DVMRP protocol outperforms ODMRP by over $200 \%$ in every case. The ability to selectively choose the next hop of a multicast route requires less overhead than the mini-broadcast ODMRP performs at every hop. Even with the additional overhead of determining the shortest route from every source to every destination, DVMRP maintains a data-to-overhead ratio that increases as more subscribers join the multicast group.

The extra overhead of ODMRP, however, pays off well when it comes to it's nearly $100 \%$ reliability. With many sources, the replication of the mesh-based architecture provides enough redundancy that nearly every packet created is successfully delivered. Similarly, the protocol is not affected by the critical satellite failure, so long as the satellite does not provide critical connectivity for a ground station to the satellite network.

The difference between the two protocols means that it is necessary to match the protocol to the system requirements. Further refining of ODMRP and DVMRP will lead to multicasting protocols that are adapted to the requirements of a LEO satellite constellation.

\section{REFERENCES}

[1] C. Perkins, "IP mobility support," RFC 2002, Oct 1996.

[2] C. Perkins, "IP encapsulation within IP," RFC 2003, Oct 1996.

[3] C Perkins, "Minimal encapsulation within IP," RFC 2004, Oct 1996

[4] J.D. Solomon, "Applicability statement for IP mobility support," RFC 2005, Oct 1996.

[5] D. Cong, M. Hamlen, and C. Perkins "The definitions of managed objects for IP mobility support using SMIv2," RFC 2006, Oct 1996.

[6] T. Pusateri "Distance vector multicast routing protocol," draft-ietf-idmr-dvmrp-v3-10.txt, Aug 2000.

[7] M. Ramalho, "Intra- and inter- domain multicasting routing protocols: a survey and taxonomy," IEEE Communications: Surveys and Tutorials, Jan-Mar 2000.

[8] J. Broch, D. Maltz, D. Johnson, Y. Hu, and J. Jetcheva, "A performance comparison of multi-hop wireless ad hoc network routing protocols," MOBICOM 1998, pp. $85-97,1998$

[9] S.J. Lee, W. Su, and M. Gerla, "On-demand multicast routing protocol (ODMRP) for ad hoc networks," draftietc-manet-odmrp-02.txt, Jan 2000.

[10] S. H. Bae, S. J. Lee, W. Su, and M. Gerla, "The design, implementation and performance evaluation of the ondemand multicast routing protocol in multi-hop wireless networks," IEEE Networks, pp. 70-77, Jan/Feb 2000.

[11] S. J. Lee, W. Su, J. Hsu, M. Gerla, and R. Bagrodia, “A performance comparison study of ad hoc wireless multicast protocols," Proceedings of IEEE Infocom 2000, 2000. 